\title{
Shifting culture and taking action to reduce smoking and premature death among people with a mental health condition
}

\author{
Katy Harker and Hazel Cheeseman
}

\author{
Katy Harker is a Registrar in \\ Public Health at Action on \\ Smoking and Health, \\ London, UK. \\ Hazel Cheeseman is a Director \\ of Policy at Action on Smoking \\ and Health, London, UK.
}

\begin{abstract}
Purpose - Mental health conditions affect almost a quarter of the population who die on average 10-20 years earlier than the general population. Smoking is the single largest cause of this gap in life expectancy. Smoking rates among people with mental health conditions have barely changed over the last 20 years during a time when rates have been steadily falling in the general population. Action is needed to address the growing difference in smoking rates among those with a mental health condition compared to the general population. The paper aims to discuss these issues.

Design/methodology/approach - This work has been informed by the input of a wide range of experts and professionals from across public health, mental health and the wider NHS.

Findings - People with a mental health condition are just as likely to want to stop smoking as other smokers but they face more barriers to quitting and are more likely to be dependant and therefore need more support. Quitting smoking does not exacerbate poor mental health; in fact the positive impact of smoking cessation on anxiety and depression appears to be at least as large as antidepressants.

Originality/value - The full report outlines the high-level ambitions and the specific actions that must be realised to drive down smoking rates among those with a mental health condition.

Keywords Tobacco, Mental health, Health policy

Paper type Brief report

Smoking is the largest cause of preventable death in England. In 2013, smoking was responsible for over 78,000 deaths, 17 per cent of all deaths in adults aged 35 and over that year (Health and Social Care Information Centre (HSCIC), 2015). Around one in four people in England experience a mental health condition in any one year, most commonly anxiety and depression (APMS, 2007). Although mental health conditions vary widely, there is long-standing evidence that smoking prevalence is substantially higher among most mental health conditions, and increases with the severity of the condition (McManus et al., 2010). It has been clear for many years that people with mental health conditions die on average 10-20 years earlier than those without (Odegard, 1951; Chesney et al., 2014; Chang et al., 2011). It is now clear that increased suicide rates are not responsible for this discrepancy but in fact it is due to socioeconomic, healthcare and clinical risk factors (Druss et al., 2011; Thornicroft, 2013) with smoking the single largest contributor to reduced life expectancy (Royal College of Physicians and the Royal College of Psychiatrists, 2013).

The issue was highlighted in the 2011 cross-government strategy, "No Health without Mental Health" (HM Government, 2011), which stated that: "we are clear that we expect parity of esteem between mental and physical health services", and the 2016 Mental Health Taskforce Report's recommendation that "physical health checks and smoking cessation programmes should be made available for everyone with a severe mental illness" (Mental Health Taskforce, 2016).
\end{abstract}

Received 22 September 2016 Accepted 23 September 2016 
In the last 20 years smoking rates for the general population have fallen significantly from around 27 per cent in the mid-90s to 19 per cent by 2014 (Office for National Statistics, 2016). During this time however, rates among those with a mental health condition have not fallen, with smoking rates estimated to be at 40 per cent throughout the last 20 years. Successive comprehensive tobacco control strategies have made a real difference to smoking rates in the general population but have had limited impact on this group, and it is clear that a more targeted approach is needed.

To address this public health crisis Action on Smoking and Health consulted with leading academics, front line staff and experts by experience and produced a report that was endorsed by 26 organisations across mental health and public health. Building on work by other organisations (Royal College of Physicians and the Royal College of Psychiatrists, 2013; Mental Health Taskforce, 2016; Public Health England, 2015) this report sets out the 12 ambitions and the specific actions needed to improve support for people with mental health conditions to reduce their smoking. The ambition of the report is that smoking in this population reduces to less than 5 per cent by 2035, the same aspiration as for the population as a whole. There is no single magic bullet to achieve this goal and the 12 ambitions set out a comprehensive approach including improving leadership, addressing the culture of mental health services in relation to smoking, ensuring effective targeted programs are available to help people to quit, putting relevant training in place for staff and developing and implementing effective harm reduction strategies are in place.

Despite high rates of smoking and levels of addiction in this population, people with mental health conditions are no less likely to want to quit smoking but they expect to find it more difficult than the general population (HSClC, 2010). Treatments to help people quit smoking that work in the general population work for those with a mental illness regardless of the severity of the illness (National Institute for Health Care and Clinical Excellence, 2013). They are equally effective, and do not worsen mental state (Banham and Gillbody, 2010; Tsoi et al., 2010). However, people with a mental health condition face significant barriers to quitting, in particular higher than average levels of addiction and being part of peer groups where smoking rates are high. As such, they are more likely to need intensive support to quit smoking and will benefit from programmes tailored to their needs. Of particular importance for this group is access to stop smoking medications and providing access to peer support, which has been shown to be effective (Ford et al., 2013; Davidson et al., 2012).

Some health professionals can have low expectations about behaviour change among people with a mental health condition, seeing it as too difficult an issue to tackle (Lawn and Condon, 2006), or not a part of their role (Kerr et al., 2013). This must change if smoking rates are to be bought down as prompts from health professionals have been shown to be an important driver in quit attempts among smokers (Stead et al., 2013).

Most people who have a mental health condition will not go into a hospital setting and will never have an inpatient stay (APMS, 2007). It is therefore necessary for all mental health services to be aware of the smoking status of service users and seek to ensure their staff are trained to treat tobacco dependence. This includes mainstream health services such as primary care where there is evidence that professionals are significantly less likely to intervene with smokers with a mental health condition than with those without (Szatkowski and McNeill, 2013).

There is a real challenge to ensuring continuity of care across diverse and fragmented services, including the provision of support to smokers to quit. What is needed are clear, joined up treatment pathways across a locality that are ambitious about reducing harm from tobacco for the whole mental health population. Relapse prevention is vital in this group, and special attention must be paid to individuals following discharge from an inpatient unit by linking them in with community services and their GP.

The quality of support to service users must be driven by commissioning and reinforced through the inspection process. Local and national drivers are needed to incentivise change in all mental healthcare settings. Additionally, when developing services for those with mental health conditions, there should be service user involvement embedded at all stages of the decision-making process.

As well as the extensive benefits from physical health of quitting smoking, there is also evidence showing benefits to mental health. For some with mental health symptoms smoking can feel like "self-medicating" and people will say that it is an important way for them to deal with stress. 
However, this relief is temporary, linked to relieving withdrawal from nicotine, and in the long run can exacerbate symptoms (McDermott et al., 2013; Taylor et al., 2014). Smoking cessation is associated with reduced depression, anxiety and stress as well as improved positive mood and quality of life compared with continuing to smoke. The impact of smoking cessation on anxiety and depression appears to be at least as large as antidepressants (Taylor et al., 2014) and treatment for tobacco dependence should always be provided alongside treatment for mental health conditions.

The full report, including all our ambitions in full, is available here: www.ash.org.uk/current-policyissues/health-inequalities/smoking-and-mental-health/the-stolen-years

\section{References}

APMS (2007), "Adult Psychiatric Morbidity in England - 2007, Results of a household survey", NHS Information Centre for Health and Social Care, 27 January, 2009.

Banham, L. and Gilbody, S. (2010), "Smoking cessation in severe mental illness: what works?", Addiction, Vol. 105 No. 7 , pp. 1176-89.

Chang, C.K. et al. (2011), "Life expectancy at birth for people with serious mental illness and other major disorders from a secondary mental health care case register in London”, PLoS One, Vol. 6 No. 5, e19590.

Chesney, E., Goodwin, G.M. and Fazel, S. (2014), "Risks of all-cause and suicide mortality in mental disorders: a meta-review”, World Psychiatry, Vol. 3 No. 2, pp. 153-60.

Davidson, L., Bellamy, C., Guy, K. and Miller, R. (2012), "Peer support among persons with severe mental illnesses: a review of evidence and experience", World Psychiatry, Vol. 11 No. 2, pp. 123-8.

Druss, B.G., Zhao, L., Von, E.S., Morrato, E.H. and Marcus, S.C. (2011), “Understanding excess mortality in persons with mental illness: 17-year follow up of a nationally representative US survey”, Med Care, Vol. 49 No. 6, pp. 599-604.

Ford, P., Clifford, A., Gussy, K. and Gartner, C. (2013), "A systematic review of peer-support programs for smoking cessation in disadvantaged groups", International Journal of Environmental Respiratory Public Health, Vol. 10 No. 11, pp. 5507-22.

Health and Social Care Information Centre (HSCIC) (2015), Statistics on Smoking, Health and Social Care Information Centre, Leeds.

Health and Social Care Information Centre (HSCIC) (2010), Health Survey for England, Health and Social Care Information Centre, Leeds.

HM Government (2011), "No health without mental health a cross-government mental health outcomes strategy for people of all ages", HM Government, London.

Kerr, S., Woods, C., Knussen, C., Watson, H. and Hunter, R. (2013), "Breaking the habit: a qualitative exploration of barriers and facilitators to smoking cessation in people with enduring mental health problems", BMC Public Health, Vol. 13 No. 221.

Lawn, S. and Condon, J. (2006), "Psychiatric nurses' ethical stance on cigarette smoking by patients: determinants and dilemmas in their role in supporting cessation", International Journal of Mental Health Nursing, Vol. 15 No. 2, pp. 111-18.

McDermott, M.S., Marteau, T.M., Hollands, G.J., Hankins, M. and Aveyard, P. (2013), "Change in anxiety following successful and unsuccessful attempts at smoking cessation: cohort study", The British Journal of Psychiatry, Vol. 202 No. 1, pp. 62-7.

McManus, S., Meltzer, H. and Campion, J. (2010), Cigarette Smoking and Mental Health in England. Data from the Adult Psychiatric Morbidity Survey 2007, National Centre for Social Research, London.

Mental Health Taskforce (2016), "The five year forward view for mental health", a report from the independent Mental Health Taskforce to the NHS in England.

National Institute for Health Care and Clinical Excellence (2013), "Smoking harm reduction", Public Health Guidelines PH45.

Odegard, O. (1951), "Mortality in Norwegian mental hospitals 1926-1941", Acta Genet Stat Med, Vol. 2 No. 2 , pp. $141-73$. 
Office for National Statistics (2016), "Opinions \& Lifestyle Survey: adult smoking habits in Great Britain", London.

Public Health England (2015), "Smoking cessation in secondary care: mental health settings", Guidance for Commissioners, London.

Royal College of Physicians and the Royal College of Psychiatrists (2013), "Smoking and mental health", A joint report by the Royal College of Physicians and the Royal College of Psychiatrists, London.

Stead, L.F., Bergson, G. and Lancaster, T. (2013), "Physician advice for smoking cessation", The Cochrane Collaboration, Vol. 5, CD000165.

Szatkowski, L. and McNeill, A. (2013), "The delivery of smoking cessation interventions to primary care patients with mental health problems”, Addiction, Vol. 108 No. 8, pp. 1487-94.

Taylor, G., McNeill, A., Girling, A., Farley, A., Lindson-Hawley, N. and Aveyard, P. (2014), "Change in mental health after smoking cessation: systematic review and meta-analysis", British Medical Journal, Vol. 348, g1151.

Thornicroft, G. (2013), "Premature death among people with mental illness: at best a failure to act on evidence; at worst a form of lethal discrimination", British Medical Journal, Vol. 346, f2969.

Tsoi, D.T., Porwal, M. and Webster, A.C. (2010), "Efficacy and safety of bupropion for smoking cessation and reduction in schizophrenia: systematic review and meta-analysis", The British Journal of Psychiatry, Vol. 196 No. 5, pp. 346-53.

\section{Further reading}

Health and Social Care Information Centre (2007), "Adult psychiatric morbidity in England", results of a household survey.

For instructions on how to order reprints of this article, please visit our website: 